\title{
Experiencias con huanglonbing en limón Mexicano en el Estado de Colima, México
}

\author{
Marciano Manuel Robles González, Mario Orozco Santos ${ }^{1}$, \\ Miguel Ángel Manzanilla Ramírez ${ }^{1}$ José Joaquín Velázquez Monreal ${ }^{1}$, \\ Víctor Manuel Medina Urrutia ${ }^{2} \&$ Eduardo Sanches Stuchi ${ }^{3}$ \\ RESUMEN
}

Las primeras detecciones del huanglongbing (HLB) en plantaciones comerciales de limón Mexicano en Colima, México, se hicieron en abril de 2010. Siete meses después se detectaron árboles con síntomas de la enfermedad en todos los municipios productores de cítricos del Estado y para el 2013 se encontró en el 100\% de los árboles de todas las plantaciones de más de dos años de edad. En las nuevas plantaciones, los árboles se infectaron rápidamente y en menos de un año todos presentaron los síntomas. El HLB afectó el vigor y redujo el potencial de rendimiento de los árboles, lo que provocó una fuerte caída de la producción de este cítrico en Colima. En plantaciones con buen manejo de riegos y fertilización, los árboles reducen su capacidad de fructificación hasta un $50 \%$, pero si el manejo nutricional es deficiente las pérdidas de rendimiento pueden superar el $80 \%$. A cinco años de la llegada del HLB a Colima, el rendimiento promedio estatal se redujo de 17,8 ton.ha ${ }^{-1}$ registrado en 2010 a solo 9,3 ton.ha ${ }^{-1}$ en 2014. A partir del 2015 se registró una recuperación constante en la producción que permitió que para 2017 este promedio suba a 13,5 ton.ha-1. Este documento presenta las primeras experiencias recabadas sobre esta enfermedad afectando plantaciones comerciales de limón Mexicano en Colima, México a ocho años de su aparición.

Términos de indexación: Citrus aurantifolia, HLB, epidemia, rendimiento, manejo agronómico, Candidatus Liberibacter spp.

\section{Experiências com o huanglonbing em limão Galego no Estado de Colima, México}

\section{RESUMO}

As primeiras detecções de huanglongbing (HLB) nos pomares comerciais de limão Galego no Estado de Colima, México foram feitas em abril de 2010. Sete meses depois, foram detectadas árvores com sintomas da doença em todos os municípios produtores de cítricos do Estado e, em 2013, a doença foi encontrada em 100\% das plantas dos pomares com mais de dois anos de idade. Nos pomares com menos de dois anos o progresso da doença foi mais rápido e em menos de um ano todas as plantas estavam contaminadas. O HLB afetou o vigor e reduziu o potencial de rendimento das árvores, o que causou uma queda acentuada na produção dessa fruta de citros em Colima.

\footnotetext{
${ }^{1}$ Instituto Nacional de Investigaciones Forestales, Agrícolas y Pecuarias, Campo Experimental Tecomán, Tecomán, Colima, México

${ }^{2}$ Centro Universitario de Ciencias Biológicas y Agropecuarias, Universidad de Guadalajara, Nextipac, Zapopan, Jalisco, México

${ }^{3}$ Estação Experimental de Citricultura de Bebedouro, Embrapa Mandioca e Fruticultura, Bebedouro, SP, Brasil

Autor de correspondencia: Eduardo Sanches Stuchi, Estação Experimental de Citricultura de Bebedouro, Embrapa Mandioca e Fruticultura, Rodovia Faria Lima, Km 384, CEP 14713-000, Bebedouro, SP, Brasil. E-mail: eduardo.stuchi@embrapa.br
} 
Em plantações com bom manejo de irrigação e fertilização, as árvores reduzem sua capacidade de frutificação em até $50 \%$, mas se o manejo nutricional é deficiente, as perdas de produtividade podem exceder $80 \%$. Cinco anos após a chegada do HLB em Colima, a produtividade média caiu de 17,8 t ha-1, registrado em 2010, para apenas $9,3 \mathrm{t} \mathrm{ha}^{-1} \mathrm{em}$ 2014. A partir de 2015, houve uma recuperação constante na produção e para 2017 essa média subiu para 13,5 t ha ${ }^{-1}$. Este artigo apresenta as observações sobre esta doença afetando as plantações comerciais de limão Galego em Colima, no México, oito anos depois de sua aparição.

Termos de indexação: Citrus aurantifolia, HLB, epidemia, rendimento, manejo agronômico, Candidatus Liberibacter spp.

\section{IMPORTANCIA ECONÓMICA Y SOCIAL DEL CULTIVO DEL LIMÓN MEXICANO}

De acuerdo con datos del Servicio de Información Agroalimentaria y Pesquera (SIAP, 2018), durante el 2017 se registraron 89,795.2 ha dedicadas al cultivo de limón Mexicano [Citrus aurantifolia (Christm) Swingle]. La producción anual de fruta fue de 1,120,972.5 ton, con un valor de 5,857.1 millones de pesos. En el estado de Colima se cultivan 18,321.9 ha con un volumen de producción de 235,545.5 ton. Esta agroindustria genera una gran cantidad de empleos para jornaleros y profesionales en el campo, los empaques, la industria, el transporte y la comercialización. Además existe un considerable número de viveros productores de plantas y de empresas proveedoras de insumos que se benefician con este cultivo (Robles-González et al., 2013).

\section{EL HUANGLONGBING}

El huanglongbing (HLB) es la enfermedad más devastadora para los cítricos a nivel mundial (Halbert \& Manjunath, 2004; Bové, 2006; Manjunath et al., 2008). No se conoce cura para los árboles infectados (Gottwald et al., 2007), los que en poco tiempo se vuelven decadentes e improductivos y en algunos casos mueren. Históricamente esta enfermedad ha sido responsable del declinamiento de las industrias de cítricos en varios países del sudeste de Asia, la Península Arábiga, las costas del Sur y el Este de África, y más recientemente se ha convertido en la principal amenaza para la sostenibilidad de la industria cítrica en Sur, Centro y Norte América (Bassanezi et al., 2013). Actualmente no se conocen fuentes de resistencia genética para el HLB dentro del género Citrus y de muchos de los géneros cercanos. Los síntomas del HLB están asociados a la presencia de una alphaproteobacteria del tipo Gram-negativa, la cual está restringida al floema de las plantas hospederas y que es conocida como Candidatus Liberibacter spp. Actualmente, se encuentra ampliamente diseminada en las áreas citrícolas del sudeste de Asia, el sur y el este de África, y la península Arábiga (Bové, 2006). Hasta principios del 2004, el continente americano era considerado como libre de HLB, pero en marzo de ese año la enfermedad fue detectada en el estado de São Paulo, Brasil. En Estados Unidos se detectó por primera vez en agosto de 2005 en el estado de Florida, posteriormente en Louisiana en 2008, Georgia en 2009, Texas y California en 2012. También se le ha reportado en Cuba, Belice, Jamaica, y otros países de la cuenca del Caribe, donde está causando fuertes daños a la citricultura (Wang \& Trivedi, 2013).

En México, la enfermedad se detectó por primera vez en julio de 2009 en árboles de limón Mexicano de traspatio de una comunidad costera del municipio de Tizimín, en el estado de Yucatán. Ese mismo año también se encontró en los estados de Quintana Roo, Nayarit y Jalisco en plantaciones comerciales de limón Mexicano y limón Persa (C. latifolia Tanaka). Durante el 2010, se hicieron nuevas detecciones que incluyeron a Campeche, Colima, Sinaloa y Michoacán. Para finales del 2012, las detecciones incluían además a Chiapas, Baja California, Hidalgo y Tabasco. Recientemente se ha informado que esta enfermedad se encuentra en 24 estados citrícolas de México (SENASICA, 2018). El HLB se ha vuelto endémico en las áreas productoras de limón Mexicano y limón Persa en los estados de Colima, Michoacán Jalisco y Nayarit, donde ha causado un impacto económico y social muy importante. Tan solo en Colima se estimó una caída en la demanda de mano de obra para labores de campo y cosecha por 2,435 empleos de tiempo completo, equivalentes a 300 jornales anuales, de 2010 a 2012 (Avalos \& González-Sánchez, 2013).

\section{SÍNTOMAS}

En campo, los primeros síntomas de HLB en limón Mexicano se pueden observar después de tres a cuatro meses después de la infección natural con psilidos. Un periodo 
muy corto si lo comparamos con lo reportado para cítricos dulces, que puede tardar hasta dos años (Gottwald, 2010; Bassanezi, 2010). Al principio, los árboles muestran uno o dos sectores de su copa, entre 0,5 a $1,0 \mathrm{~m}^{2}$, con apariencia clorótica, dando un aspecto sectorizado de la enfermedad, tal como lo consigna Bové (2006) para otros cítricos. En los siguientes cuatro a cinco meses, el número y tamaño de los sectores sintomáticos en esos árboles se incrementa significativamente llegando a cubrir toda la copa. La velocidad con que la enfermedad cubre la copa de los árboles está estrechamente relacionada a la cantidad de psilidos infectivos en la plantación o en plantaciones vecinas. En plantaciones con pobre manejo de plagas las poblaciones del psilido son altas y en consecuencia las reinfecciones también son altas.

Las hojas en sectores sintomáticos presentan manchas irregulares de color amarillento. En algunos casos además se puede observar acorchamiento de nervaduras. En esta especie los síntomas de moteado son muy evidentes y fáciles de identificar, motivo por el cual las detecciones en campo tienen hasta $95 \%$ de coincidencias con las pruebas con PCR tiempo real. Los brotes vegetativos que crecen a partir de ramas con síntomas de HLB, desde que emergen hasta que completan su desarrollo y alcanzan su madurez, presentan aspecto vigoroso, sin síntomas del HLB y su color es verde claro, muy similar a lo observado en brotes vegetativos de la misma edad, pero que se desarrollaron en árboles sanos. Al madurar los brotes, las hojas desarrollan el moteado amarillento con distribución asimétrica, característico del HLB (Robles-González et al., 2010). Los sectores sintomáticos de un árbol afectado por HLB presentan follaje escaso y fuerte reducción en la producción de fruta (Figura 1A). En contraste, los sectores asintomáticos tienen semejanza con los árboles sanos con follaje verde y mayor fructificación (Figura 1B). Después de ocho años de estar presente la enfermedad del HLB en los árboles de limón Mexicano, no se ha observado síntomas de maduración invertida, deformación o caída prematura de fruta, que puedan estar relacionados con el HLB, como lo señala Bové (2006) y Bassanezi (2010) para cítricos dulces.

Los frutos cosechados en sectores sintomáticos presentan un tamaño ligeramente menor, comparado con los frutos provenientes de sectores asintomáticos del mismo árbol o de árboles sanos. Este resultado coincide con lo señalado por Bassanezi et al. (2011), quien concluye que la enfermedad tiene un efecto negativo sobre el tamaño de la fruta, solamente en los sectores sintomáticos de la copa de los árboles. La enfermedad no afectó significativamente el grosor de cáscara, el número de semillas, el contenido de semillas abortadas, el porcentaje de jugo, la acidez, ni los valores de ${ }^{\circ}$ Brix (Robles-González et al., 2013). Por lo que la calidad química de la fruta y por lo tanto la aceptación del consumidor no se vieron comprometidas. Esto difiere con los resultados obtenidos por Bassanezi et al. (2009) en naranjas, donde la enfermedad reduce significativamente el contenido de jugo y el total de sólidos solubles (TSS), pero además incrementa la acidez.
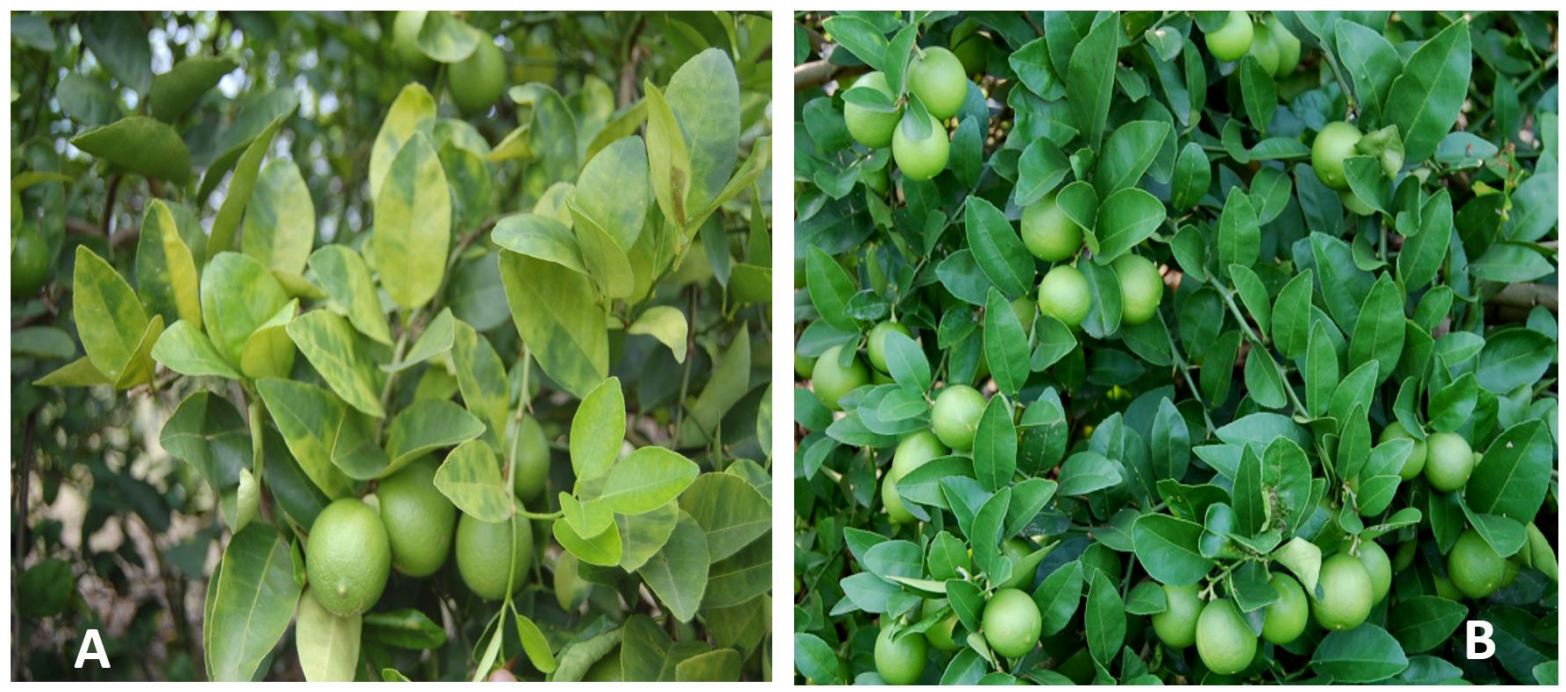

Figura 1. (A) sector de árbol mostrando síntomas característicos del HLB en las hojas y con escasa producción de fruta; (B) Sector sano con follaje denso, de color verde característico de la variedad y buena fructificación. 
En otros cítricos, como naranja y toronja, se ha determinado que el HLB afecta fuertemente los tejidos del floema (Folimonova \& Achor, 2010), lo que causa problemas para la translocación de asimilados en las plantas hospederas. Estudios a nivel genómico han podido determinar que el HLB puede alterar la expresión de más 600 genes de la planta infectada (Kim et al., 2009; Liao \& Burns, 2012; Mafra et al., 2013; Zheng \& Zhao, 2013). Los genes cuya expresión es alterada están relacionados principalmente con sistemas de defensa de la planta (Kim et al., 2009), el metabolismo de sacarosa y almidón, síntesis de hormonas y de señalización (Martinelli et al., 2012). Esto puede explicar los síntomas en hojas, la perdida de vigor en los árboles y su consecuente reducción en el rendimiento de fruta que se observa en limón Mexicano.

\section{DISEMINACIÓN}

Las bacterias asociadas a los síntomas de la enfermedad del HLB son diseminadas de manera natural entre árboles por insectos vectores. $C a$. Liberibacter asiáticus (CLas) y $C a$. Liberibacter americanus (CLam) son transmitidas por el psílido asiático de los cítricos (PAC) (Diaphorina citri Kuwayana) en Asia y América. Por su parte, $\mathrm{Ca}$. Liberibacter africanus (CLaf) es transmitido por Trioza erytreae (Del Guercio) (Bové, 2006). La variante $C a$. Liberibacter americanus (CLca) también es diseminada por el PAC (ICA, 2010; Manjunath et al., 2015). Otra manera en que se transmiten estas bacterias es mediante injerto cuando se usan yemas o varetas infectadas. Sin embargo, la movilización a grandes distancias de muchos fitopatógenos se hace por el transporte de plantas o varetas infectadas que realiza el hombre, principalmente cuando la movilización de materiales es de manera clandestina y no se cumplen los protocolos fitosanitarios. No se ha demostrado que la enfermedad se transmita por la semilla a las plantas que de ellas se originan (Bové, 2006; Hilf, 2011). En Colima la diseminación del HLB dentro y entre huertas fue muy rápida debido a las condiciones especiales que se presentan en las zonas productoras de cítricos, entre ellas el clima cálido y la aplicación de riegos que promueve la constante emisión de brotes vegetativos en los árboles de limón Mexicano durante todo el año, que a su vez favoreció el desarrollo de abundantes poblaciones del PAC. Además de lo anterior se agregó el hecho de que no se hizo la eliminación oportuna de los primeros árboles infestados.

\section{HOSPEDEROS}

Todos los cultivares de cítricos, son susceptibles en algún grado al HLB. Se ha observado que la naranja dulce, los pomelos y mandarinas son más susceptibles, mientras que algunos limones verdaderos y las limas ácidas son menos sensibles (Graça, 1991; Folimonova et al., 2009). Por su parte Stover et al. (2010) señala que que el género Poncirus trifoliata Raf. y algunos de sus híbridos pueden tolerar y/o suprimir al patógeno del HLB de manera significativa y en consecuencia presentar los mayores niveles de tolerancia. Esta bacteria se ha observado afectando también otros géneros como: Microcitrus, Swinglea, Atalantia, Clausena, Limonia, Balsamocitrus, Aeglopsis, Severinia, y Murraya. Experimentalmente, también se ha logrado inducir síntomas en Periwinkle o teresita (Catharanthus roseus L. G. Don.) y tabaco (Nicotiana tabacum L. cv Xanthi), dos hospederos que no son Rutáceas (Knapp et al., 2014; Abdullah et al., 2009; Halbert \& Manjunath, 2004).

\section{EL HLB EN PLANTACIONES COMERCIALES LIMÓN MEXICANO EN COLIMA}

\section{Características de las plantaciones de limón en Colima}

En Colima, las áreas productoras más importantes presentan características de clima cálido subhumedo $\mathrm{A}(\mathrm{w} 0)$ y cálido semiseco (BS1), con lluvias en verano. La temperatura media anual de $26,0^{\circ} \mathrm{C}$, precipitación pluvial que va de $\operatorname{los} 450$ a 786,3 mm anuales y que se concentra en los meses de julio a septiembre. Bajo estas condiciones las plantaciones de limón requieren aplicación de riego la mayor parte del año. El clima cálido de la región, junto con la aplicación periódica de riegos, crean las condiciones propicias para que los árboles emitan brotes vegetativos y florales de manera casi continua la mayor parte del año. Este ambiente, con suficiente tejido vegetativo tierno y suculento, es favorable para la reproducción del PAC, lo que propicia que se mantengan poblaciones altas de este insecto prácticamente durante todo el año. Un estudio sobre la bioecología del PAC realizado durante 2008, permitió determinar que el insecto estaba presente durante todo el año y que sus poblaciones más abundantes estaban asociadas a la presencia de flujos importantes de brotación vegetativa en los árboles de limón mexicano, debido a que en esos órganos es donde las hembras ovipositan y 
sus estados inmaduros se desarrollan. Los adultos pueden estar presentes alimentándose de brotes tanto tiernos como maduros (Robles-González et al., 2010).

\section{Primeras detecciones y diseminación regional del HLB}

La primera detección oficial del HLB en el estado de Colima fue realizada por personal del Comité Estatal de Sanidad Vegetal de Colima (CESAVECOL) el 7 de abril de 2010 en una plantación comercial de limón mexicano de cuatro años de edad, ubicada muy cerca de la playa (Figura 2A). Los cinco árboles diagnosticados positivos al HLB presentaban uno o dos sectores de su copa donde se apreciaban ramas de entre 8 y $10 \mathrm{~mm}$ de diámetro y de 1,0 a 1,2 m de longitud, con hojas sintomáticas en toda su extensión, excepto en el brote vegetativo terminal, lo que hace suponer que los primeros síntomas de HLB estuvieron presentes en este predio tres a cuatro meses antes de su detección y que la infección primaria pudo ocurrir con anterioridad. Este tiempo fue suficiente para que algunos psílidos infectivos ya hubieran diseminado la enfermedad a otros árboles del mismo huerto y hacia árboles de los huertos vecinos. En ese predio se aplicaba el manejo integrado de plagas con bajo uso de insecticidas por lo que en los árboles sintomáticos se podía apreciar brotes vegetativos tiernos siendo infestados con ninfas y adultos del PAC. Un mes después se detectaron
24 árboles sintomáticos adicionales en este predio y al mismo tiempo se detectó la enfermedad en dos huertos cercanos. Tres meses después se hicieron detecciones en huertas de los municipios de Armería y Manzanillo, a $30 \mathrm{Km}$ del predio donde se hizo la primera detección de la enfermedad, lo que confirma que el psílido había tenido suficiente tiempo para movilizar al patógeno. Para el séptimo mes, en su reporte mensual el CESAVECOL (2011) informó que ya se habían detectado árboles positivos HLB en todas zonas productoras de limón mexicano en el estado de Colima (Figura 2B).

El patrón de dispersión observado en Colima tiene fuerte coincidencia con lo descrito tanto en Brasil (Bassanezi, 2010) como en Florida E.U.A (Irey, 2010), donde en poco tiempo la enfermedad se detectó en un gran número de municipios o condados, sin embargo, la velocidad de dispersión observada en Colima parece ser más rápida y puede explicarse por las poblaciones abundantes del PAC en la región. En julio de 2013 se muestrearon 7250 árboles en 290 parcelas ubicadas en 24 módulos de asesoría técnica, distribuidas en todas las zonas productoras del Estado. El muestreo demostró que el HLB se encontraba afectando el 100\% de los árboles de todas las parcelas de los 24 módulos. Para esta fecha los árboles tenían un promedio de 50\% de sus copas afectas por la enfermedad y presentaban ya fuerte afectación en su rendimiento de fruta (Robles-González et al., 2013).
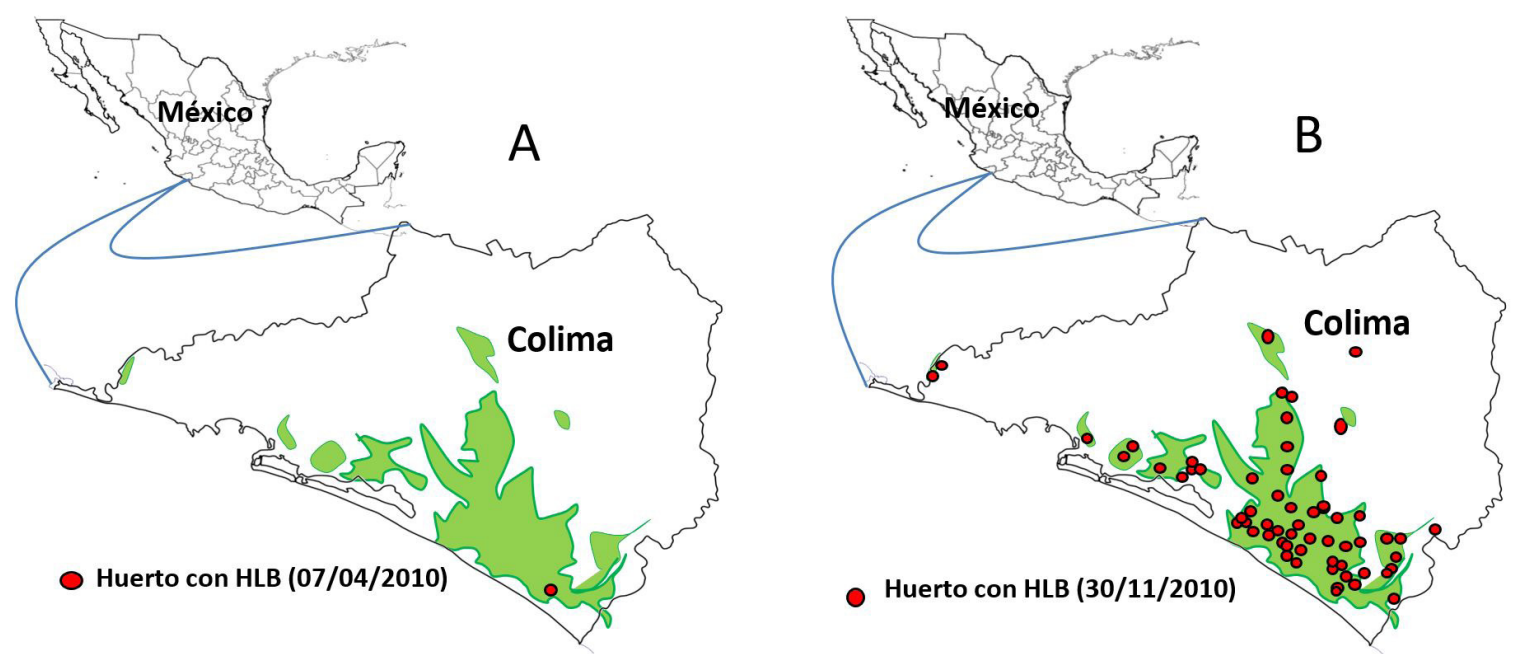

Figura 2. Áreas con plantaciones comerciales de limón mexicano en el estado de Colima (verde). (A). Punto donde se detectaron los primeros árboles con HLB y (B). Puntos donde ya se habían detectado árboles positivos siete meses después (B). 


\section{Diseminación del HLB dentro de huertos}

A nivel de huerto la epidemia de HLB presentó dos dinámicas de infección. La 'infección inicial', producida por psilidos infectivos que recién arribaron a la huerta y que provenían de otros huertos afectados por HLB. Con esta infección, los árboles sintomáticos normalmente van apareciendo dispersos por toda la plantación con distribución aleatoria. Por su parte, las 'infecciones secundarias' son las causadas de manera repetida por psilidos que se reproducen en árboles enfermos dentro de la misma plantación. En este caso, los árboles sintomáticos van apareciendo formando agregados a partir de los primeros árboles detectados con la enfermedad. Las infecciones secundarias se presentan en plantaciones que no aplican medidas de control del PAC, lo que permite que el

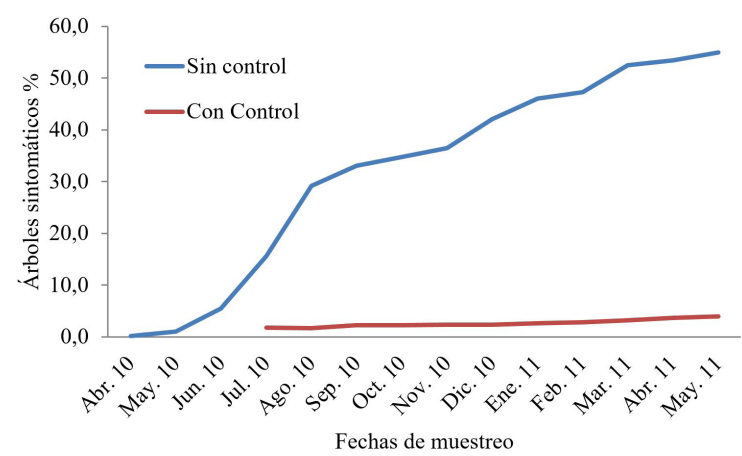

Figura 3. Dinámica del porcentaje acumulado de la detección de árboles con síntomas de HLB en dos huertos limón mexicano con distinto manejo de plagas al inicio de la epidemia. insecto se reproduzca en árboles enfermos y trasmita rápidamente la bacteria a sectores sanos del mismo árbol y hacia los árboles vecinos. Por lo tanto, la dinámica de diseminación del HLB dentro de los huertos varía en función del manejo de las poblaciones del PAC y la cantidad de insectos infectivos en la región. En el 2010, al inicio de la epidemia y cuando la cantidad de psilidos infectivos en la región aún era baja, en una plantación con 5.743 árboles, donde se aplicaba de manera alternada, insecticidas convencionales y aceite mineral, se detectaron solo 5\% de árboles sintomáticos en el trascurso de un año. En contraste, en un huerto cercano, con 2.908 árboles y donde se hizo deficiente y casi nulo control de plagas, en el mismo periodo de tiempo el 56,5\% de árboles fueron detectados con la enfermedad (Figura 3).

Esta dinámica cambió en los años siguientes, debido a que el número de árboles sintomáticos distribuidos en la región se incrementó significativamente y con ello la velocidad de diseminación de la enfermedad. Para el mes de mayo de 2011, doce meses después de la primera detección el CESAVECOL ya había localizado cerca de 10.000 árboles afectados por HLB en 627 plantaciones comerciales de cítricos en las distintas zonas productoras del Estado. La mayoría de esos árboles no fueron eliminados y se convirtieron en fuentes de inoculo. Esto fue factor importante para el incremento en los porcentajes de psílidos infectivos, como lo muestra la Figura 4A, generada con resultados del monitoreo de adultos del PAC (Félix-Portillo et al., 2011) y en consecuencia las tasas de infección en la región se elevaron a partir del este tiempo. Esto se reflejó en la dinámica de infección registrada durante 2010 y 2011 en un lote experimental
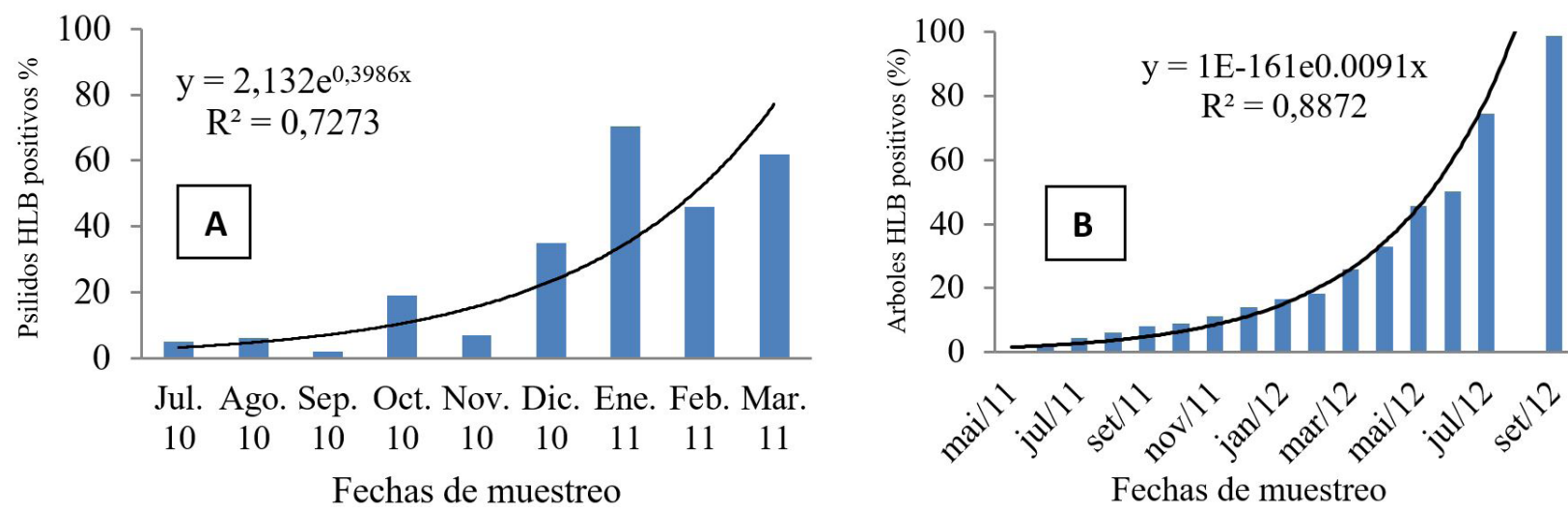

Fechas de muestreo

Figura 4. (A) Porcentajes adultos del PAC infectivos en muestreos mensuales de julio 2010 a marzo 2011 (Félix-Portillo et al., 2011); (B) Porcentaje acumulado de árboles con síntomas de HLB en muestreos mensuales de mayo de 2011 a septiembre de 2012 en un huerto con control del psílido asiático (Robles-González et al., 2013). 
con 1.174 árboles de limón mexicano, donde se aplicó un programa de control de plagas, con especial énfasis sobre el PAC, no obstante en solo 17 meses se alcanzó el 100\% de árboles infectados (Figura 4B). En este caso, la mayoría de los árboles sintomáticos se presentaron de manera dispersa y con distribución aleatoria en la plantación, lo que sugiere se debieron a infecciones principalmente primarias. Después del 2011 en las nuevas plantaciones las detecciones inician desde los tres meses después del establecimiento de los árboles y en el lapso de un año se alcanza el $100 \%$ de árboles infectados, sobre todo si no se hace el control del PAC y con ello se permiten las infecciones secundarias.

\section{Manejo de la epidemia de HLB en Colima}

En seguimiento a la normatividad establecida en el "Acuerdo por el que se dan a conocer las medidas fitosanitarias que deberán aplicarse para el control del Huanglongbing (Candidatus liberibacter spp.) y su vector" publicada por Secretaría de Agricultura, Ganadería, Desarrollo Rural, Pesca y Alimentación (SAGARPA) en el Diario Oficial de la Federación el 16 de agosto de 2010, el CESAVECOL en coordinación con los productores, la Delegación de SAGARPA en Colima y los H. Ayuntamientos de Tecomán y Armería, lograron que se eliminaran 495 árboles de limón mexicano detectados como sintomáticos en plantaciones comerciales, además de la destrucción de 972 plantas de mirtos o limonarias (Murraya paniculata L. Jack) en zonas urbanas y de 1,402,529 plantas de cítricos producidas en viveros certificados que aún estaban a cielo abierto (CESAVECOL, 2011), por desgracia esto se realizó después de que la enfermedad se había diseminados. En este acuerdo además se especificaba que el manejo fitosanitario de los huertos comerciales para producir fruta de cítricos, debería ser realizado por los productores, conforme a las siguientes recomendaciones: I) Utilizar planta proveniente de viveros certificados para replantes o establecimiento de nuevas plantaciones; II) Control del PAC, que incluya la aplicación de productos químicos autorizados; III) Realizar al menos cuatro monitoreos por año para la detección del HLB; IV) Eliminar, en un plazo de cinco días naturales, todos los árboles con síntomas de HLB que sean detectados en los monitoreos y V. Eliminar, en un plazo de cinco días naturales, la totalidad de los árboles de la huerta cuando en un muestreo se detecte que al menos, el 28\% de las plantas presentan síntomas de HLB.
Desafortunadamente entre la detección y confirmación de los primeros cinco árboles positivos al HLB en Colima y su consiguiente eliminación, se llevó bastante tiempo y no se logró la eliminación oportuna de las fuentes de infección, como lo marca el citado acuerdo. Por lo anterior, no se pudo evitar la dispersión de la enfermedad por todas las zonas productoras de cítricos en el Estado. Además de lo anterior, los productores no se mostraron dispuestos a eliminar árboles sintomáticos de sus plantaciones. Como consecuencia de ello, en las detecciones posteriores ya no se insistió en la eliminación de los árboles afectados por HLB. Tampoco se pudo evitar que se siguiera movilizando y vendiendo plantas producidas en viveros a cielo abierto y que operaban sin registro ante las autoridades fitosanitarias. Otro factor que facilito el desarrollo de la epidemia de HLB es el hecho de que en la región no se hacía control intensivo de plagas, por lo tanto muy pocos productores implementaron el control eficiente del PAC.

A partir de 2012 el SENASICA estableció un programa de control regional del psílido asiático, en base al "Protocolo para establecer Áreas Regionales de control del Huanglongbing y el Psílido Asiático de los Cítricos (ARCOs)" preparado por Robles-García (2012). Ese programa se concentró fuertemente en el combate del PAC, para lo cual la SAGARPA proporcionó insecticidas y aceites minerales, sin embargo para el caso de Colima no cubría el $100 \%$ de las plantaciones por lo que su impacto sobre las poblaciones del vector no fue significativo. Por otra parte, dicho programa no se pudo aplicar de forma adecuada debido a que no se consideraron algunos factores importantes, entre ellos, el hecho de que no todos los productores estuvieron lo suficientemente concientizados de la gravedad del problema, por lo que no participaron activamente. No se aplicaron metodologías eficientes y ágiles para la convocatoria y entrega oportuna de productos insecticidas a los productores. Además y muy importante, pocos productores contaban con equipo adecuado para la aplicación eficiente de los productos insecticidas. Debido a todo lo antes expuesto, las aspersiones no se pudieron hacer de manera sincronizada y en un tiempo menor a las dos semanas, lo que le permitió a los psílidos movilizarse entre huertos durante las aplicaciones y que las poblaciones del insecto se recuperaran rápidamente. Por otra parte, en la región existían huertos abandonados que mantenían árboles de limón sin manejo agronómico, así como una gran cantidad de árboles de diferentes cítricos y de limonaria en calles, parques y patios de casas, que se convirtieron en refugios para el PAC y fuentes permanentes de inoculo del HLB. Con base a estudios de 
caracterización agronómica de la enfermedad sobre árboles de limón mexicano en la región, que han demostrado que este cítrico tiene un nivel de tolerancia alto que le permite sobrevivir y seguir produciendo fruta, el grupo técnico del HLB de Colima promovió el manejo integrado del cultivo de limón mexicano, poniendo especial énfasis en la aplicación suficiente y oportuna de riegos y fertilización, lo que ha permitido que muchas plantaciones pudieran mantener rendimientos anualizados de 18 a 20 ton.ha ${ }^{1}$, lo que equivale a cerca del $50 \%$ de su potencial expresado antes del HLB.

\section{DIFUSIÓN Y CONCIENTIZACIÓN}

Desde el año 2008, dos años antes de la llegada del HLB, el Instituto Nacional de Investigaciones Forestales, Agrícolas y Pecuarias (INIFAP), el CESAVECOL y la FUNDACIÓN PRODUCE COLIMA AC., realizaron foros técnicos, cursos de capacitación para técnicos y productores en las distintas localidades productoras de cítricos del estado. También se tuvo constante participación en estaciones de radio locales, se entregaron folletos, se realizaron pinta de bardas, entre otras actividades, cuyo fin fue difundir el conocimiento existente sobre el HLB y su vector. Aunque se hiso bastante trabajó de difusión no fue suficiente para lograr el objetivo fundamental de concientizar a productores, empacadores y procesadores de la fruta de limón, sobre la gravedad del problema. La primera reacción de los productores y empacadores fue la negación y resistencia para implementar medidas de control de la enfermedad. Por lo tanto, no se pudieron implementar las acciones de: eliminación inmediata de árboles sintomáticos, el control eficiente del psílido asiático y uso de plantas certificadas libres del patógeno, que son los tres pilares fundamentales en el manejo del HLB.

\section{EFECTO DEL HLB SOBRE RENDIMIENTO DE FRUTA}

En campo, durante el tiempo que se establece la epidemia la, enfermedad del HLB se presenta de manera sectorizada en la copa de los árboles en limón mexicano. Los árboles pueden presentar diferente cantidad y tamaño de sectores afectados por la enfermedad, dependiendo del tiempo trascurrido desde la aparición de los primeros síntomas, así como la presencia y abundancia de psilidos infectivos en la plantación. Los sectores del árbol que manifiestan síntomas de la enfermedad tienen follaje amarillento y producen menor cantidad de fruta, comparado con los sectores con apariencia sana del mismo árbol. Por lo tanto, durante el desarrollo de la epidemia, la capacidad de producción del árbol se va reduciendo principalmente por la proporción del área de copa que está siendo afectada por HLB, tal como lo señalan Bassanezi et al. (2013). Esto se pudo demostrar al cuantificar el número de frutos a madurez de cosecha en árboles de limón mexicano con distinta proporción del área de copa mostrando síntomas del HLB (Tabla 1).

Mientras que en los árboles que aún tenian apariencia sana se registraron promedios de hasta 60,3 frutos. $\mathrm{m}^{-2}$, de copa, la cantidad de fruta fue disminuyendo conforme los árboles tenían mayor proporción de copa sintomática, hasta un mínimo de 27,6 frutos. $\mathrm{m}^{-2}$ en árboles que mostraban entre 75 y $100 \%$ de copa afectada por la enfermedad. En un lote experimental donde se evaluaba el comportamiento productivo de las variedades Lise y Colimex de limón mexicano, también se pudo observar el impacto del HLB sobre el rendimiento de fruta (Tabla 2). En este lote, los primeros cuatro árboles con síntomas se detectaron en mayo de 2011. En ese año, se registraron rendimientos de 48 y 42,0 ton.ha ${ }^{-1}$ de fruta para Lise y Colimex respectivamente. Para el 2013 el 100\% de los árboles presentaba los síntomas del HLB con porcentajes de copa afectada que superaron el $50 \%$, lo que les provocó una ligera reducción del rendimiento en ambas variedades. Sin embargo, para el 2014 la mayoría de los árboles tenían el $100 \%$ de sus copas afectadas por la enfermedad y en consecuencia los rendimientos bajaron drásticamente hasta solo 23,4 y 22,9 ton.ha ${ }^{-1}$ respectivamente. Esto representa casi un $50 \%$ de las 40 ton. ha $^{-1}$ esperadas en árboles sanos de esa edad.

Tabla 1. Número de frutos por metro cuadrado en árboles con distinto porcentaje del área de copa afectada por el HLB

\begin{tabular}{cccc}
\hline Área de copa con & Arboles & \multicolumn{2}{c}{ frutos.m $\mathrm{m}^{-2}$ de copa } \\
\cline { 3 - 4 } HLB & muestreados & No.) & $(\%)$ \\
$(\%)$ & No.) & & \\
\hline Asintomáticos & 140 & $60,3 \mathrm{a}$ & 100,0 \\
$10-25$ & 140 & $48,7 \mathrm{~b}$ & 80,8 \\
$25-50$ & 140 & $45,8 \mathrm{~b}$ & 75,9 \\
$50-75$ & 140 & $39,6 \mathrm{c}$ & 65,7 \\
$75-100$ & 140 & $27,6 \mathrm{~d}$ & 45,8 \\
\hline
\end{tabular}

Promedios con letra distinta dentro de columnas difieren estadísticamente (Tukey, $\mathrm{P}=0,05$ ). 
Tabla 2. Rendimiento anual de fruta (ton $\mathrm{ha}^{-1}$ ) en dos variedades de limón mexicano en seis años de cosechas y con afectación del HLB a partir de 2011

\begin{tabular}{crcccccc}
\hline \multirow{2}{*}{ VARIEDAD } & 2008 & 2009 & 2010 & 2011 & 2012 & 2013 & 2014 \\
\cline { 2 - 8 } & $9,7 \mathrm{a}$ & $25,6 \mathrm{a}$ & $42,6 \mathrm{a}$ & $48,7 \mathrm{a}$ & $39,3 \mathrm{a}$ & $37,9 \mathrm{~b}$ & $22,9 \mathrm{a}$ \\
Lise & $13,3 \mathrm{a}$ & $27,8 \mathrm{a}$ & $37,5 \mathrm{a}$ & $42,0 \mathrm{a}$ & $39,0 \mathrm{a}$ & $43,9 \mathrm{a}$ & $23,4 \mathrm{a}$ \\
\hline
\end{tabular}

Promedios con letra distinta dentro de columnas difieren estadísticamente (Tukey, $\mathrm{P}=0,05$ ).

El impacto de la enfermedad sobre la capacidad productiva de los árboles se reflejó en el rendimiento promedio del estado de Colima. Hasta antes de la llegada del HLB el promedio estatal superaba las 20 ton.ha ${ }^{1}$.año. A partir del 2011, esta cantidad fue bajando año con año, hasta solo 9,3 ton.ha1.año-1 registrada en el 2014 (SIAP, 2015). A partir del 2015 se ha registrado una recuperación constante en la producción que permitió que para 2017 este promedio suba a 13,5 ton.ha-1 (SIAP, 2018). El HLB ha impactado otras características agronómicas de los árboles de limón mexicano. Ha provocado cambios en la dinámica de su fenología y en la actualidad prácticamente no se observan flujos de floración abundantes como ocurría antes de la llegada de esta enfermedad a la región. Por otro lado, a finales de 2012, se observó un incremento en la incidencia de muerte de ramas en los árboles en la mayoría de las huertas en el estado. La pérdida de ramas vino a agravar la baja en los rendimientos de fruta de las plantaciones ya que en poco tiempo se puede perder hasta el 50\% de la copa del árbol y con ello la producción de fruta. En algunos casos, la pérdida de ramas propicia que el sol penetre y queme de la corteza del tronco, lo que puede llevar a la muerte del árbol. Este problema se observa en plantaciones de más de tres años de edad, donde los árboles están en plena etapa de fructificación. Árboles pequeños y en desarrollo no manifiestan este síntoma. Los estudios realizados al respecto indican que el hongo Lasiodiplodia sp. está asociado a este problema (Orozco-Santos et al., 2013).

\section{OCHO AÑOS DE APRENDIZAJE}

A ocho años desde la primera detección del HLB en huertas comerciales de limón mexicano en Colima, México, mediante trabajos de investigación y constante retroalimentación con productores, se han generado valiosas experiencias y aprendizajes. Hasta el 2010 no se tenía idea de cómo sería una epidemia del HLB en limón mexicano. Se esperaba que la enfermedad matara los árboles o los volviera totalmente improductivos en poco tiempo y se perdiera el cultivo de este cítrico en la región, como se reportó para otros cítricos en otras regiones citrícolas del mundo. La experiencia ganada hasta ahora indica que la enfermedad tiene un comportamiento diferente en limón mexicano, los árboles aunque pierden vigor, pueden seguir produciendo fruta, lo que permite convivir con la enfermedad. Está claro que solo cuando se viven experiencias con HLB, se aprende de esta enfermedad. En la región productora de Colima la relación limón mexicano y el binomio PAC y HLB es un caso muy especial. En esta región, con un clima cálido y donde los árboles emiten brotación vegetativa de manera casi constante, generan el ambiente propicio para que se presenten poblaciones abundantes de este insecto durante todo el año, lo que hace prácticamente imposible evitar la rápida diseminación de la enfermedad, que pronto se convirtió en epidemia, con sus efectos devastadores. En el proceso de la epidemia de HLB se observaron tres escenarios distintos El 'escenario uno' se inició con el arribo del $D$. citri a la región productora de cítricos de Colima. Este fue el primer aviso de una potencial epidemia. En este tiempo se implementaron algunas acciones principalmente de control legal, que tenían el objetivo fundamental el evitar que CLas se introdujera a la región. En este tiempo se hicieron trabajo de difusión con el fin de concientizar a los productores, asesores, empacadores, industrializadores y autoridades, sobre el riesgo que representaba esta enfermedad. El 'escenario dos' se inició cuando se detectaron los primeros árboles sintomáticos y se confirmó la presencia de la bacteria causante del HLB. Las acciones que se intentaron en este periodo estuvieron enfocadas a evitar que la bacteria de diseminara hacia otras plantaciones en la región. Para lograr este objetivo se recomendó la rápida eliminación de los focos de infección iniciales, lo que implicaba derribar todos los árboles sintomáticos tan pronto fuesen detectados. También se recomendó hacer el control eficiente del psílido asiático para reducir sus poblaciones y su potencial de movilizar la bacteria. Se recomendó no usar planta proveniente de 
viveros a cielo abierto por lo que a partir de esta fecha muchos viveristas tuvieron que desarrollar sus procesos de producción de plantas dentro de estructuras protegidas. A pesar de esas recomendaciones, no se pudo evitar que CLas se introdujera a la región y tampoco se pudo evitar que se diseminara a todas las zonas productoras de cítricos en el estado y por lo tanto, no se pudo evitar la epidemia del HLB. En consecuencia, se arribó al 'escenario tres'. En estas circunstancias ya no es factible la erradicación de árboles enfermos por lo que se optó por la estrategia de convivencia-sobrevivencia. Para ello se recomiendo aplicar la tecnología de manejo integrado del cultivo, mejorar la aplicación de riego y fertilización, con el fin de mantener hasta donde fuera posible el vigor y la productividad de los árboles. Si se toma en cuenta el axioma de la fitopatología que dice "si la velocidad de erradicación es mayor a la velocidad de diseminación, la erradicación es una opción viable”. En Colima la erradicación no es viable. Tomando en cuenta la experiencia vivida con HLB en Colima, se puede concluir que la mejor estrategia que se puede utilizar contra esta enfermedad, es aplicar medidas eficientes para mantener al patógeno lo más lejos posible de nuestras regiones productores. Hasta hoy aún es poca la información sobre HLB en plantaciones comerciales de limón mexicano y será necesario seguir investigando para tener mejor conocimiento del problema y desarrollar alternativas eficientes para su manejo.

\section{CONCLUSIONES Y PERSPECTIVAS}

Las experiencias en estos años de convivir con la enfermedad del HLB en plantaciones comerciales de limón mexicano demuestran que los árboles tienen un nivel de tolerancia relativamente alto, que les permite además de sobrevivir, producir cerca del $50 \%$ de fruta, comparado con un árbol sano hasta por ocho a diez años, lo cual aunque es bajo, el repunte de los precios, hacen que su cultivo sea rentable. Después de la epidemia de HLB la región productora de Colima, se está escribiendo una nueva historia para el limón mexicano en la región. Las nuevas plantaciones de este cítrico tendrán que sufrir cambios sustanciales, que se caracterizaran por la implementación de un manejo integrado y sustentable del cultivo, donde será fundamental la optimización de la nutrición de los árboles, para mantener su vigor y mejorar su respuesta a la enfermedad. El uso de mayores densidades de plantación permitirá compensar las pérdidas de rendimiento individual de los árboles y recuperar la productividad de las plantaciones. El control integrado de plagas y enfermedades coadyuvarán a mantener buenos rendimientos y alta calidad de la fruta. Esta estrategia de convivencia ayudara a ganar el tiempo suficiente mientras se generen nuevas variedades con mayor tolerancia a la enfermedad.

\section{REFERENCIAS}

Abdullah, T. L., Shokrollah, H., Sijam, K., \& Abdullah, S. N. A. (2009). Control of Huanglongbing (HLB) disease with reference to its occurrence in Malaysia. African Journal of Biotechnology, 8(17), 4007-4015.

Avalos, V. O., \& González-Sánchez, R. F. (2013). El impacto social, y económico en el comercio del HLB en la cadena de limón Mexicano en Colima. In J. J. Velázquez-Monreal, M. A. Manzanilla Ramírez, M. M. Robles-González, \& M. Orozco Santos (Eds.), Memoria del IX Simposio Internacional Citricola y ler Simposio Internacional sobre HLB en Cítricos Ácidos (pp. 1-30). Tecomán: INIFAP.

Bassanezi, R. B. (2010). Epidemiology of huanglongbing and its implications on disease management. In Memorias del $2^{\circ}$ Taller Internacional sobre el Huanglongbing y el Psílido Asiático de los Cítricos. Mérida: SAGARPA.

Bassanezi, R. B., Belasque Junior, J., \& Montesino, L. H. (2013). Frequency of symptomatic trees removal in small citrus blocks on citrus huanglongbing epidemics. Crop Protection, 52, 72-77. http://dx.doi.org/10.1016/j. cropro.2013.05.012.

Bassanezi, R. B., Montesino, L. H., \& Stuchi, E. S. (2009). Effects of huanglongbing on fruit quality of sweet orange cultivars in Brazil. European Journal of Plant Pathology, 125(4), 565-572. http://dx.doi.org/10.1007/ s10658-009-9506-3.

Bassanezi, R. B., Montesino, L. H., Gasparoto, M. C. G., Bergamin Filho, A., \& Amorim, L. (2011). Yield loss caused by huanglongbing in different sweet orange cultivars in São Paulo, Brazil. European Journal of Plant Pathology, 130(4), 577-586. http://dx.doi.org/10.1007/ s10658-011-9779-1.

Bové, J. M. (2006). Huanglongbing: a destructive, newlyemerging, century-old disease of citrus. Journal of Plant Pathology, 88(1), 7-37. 
Comité Estatal de Sanidad Vegetal en el Estado de Colima - CESAVECOL. (2011). Campaña contra el huanglonbing de los citricos (HLB): acciones y resultados: reportes mensuales del periodo abril de 2010 al 28 mayo 2011. Tecomán: CESAVECOL.

Félix-Portillo, M., Martínez-Quintana, J. A., \& ArredondoBernal, H. (2011). Estacionalidad de la infectividad del HLB (huanglonbing) en colima. In Memorias del $2^{\circ}$ Simposio Nacional sobre Investigación para el Manejo del Psílido Asiático de los Cítricos y el Huanglongbing en México. Montecillo: INIFAP.

Folimonova, S. Y., \& Achor, D. S. (2010). Early events of citrus greening (huanglongbing) disease development at the ultrastructural level. Phytopathology, 100(9), 949-958. http://dx.doi.org/10.1094/PHYTO-100-9-0949.

Folimonova, S. Y., Robertson, C. J., Garnsey, S. M., Gowda, S., \& Dawson, W. O. (2009). Examination of the responses of different genotypes of citrus to Huanglongbing (citrus greening) under different conditions. Phytopathology, 99(12), 1346-1354. http://dx.doi.org/10.1094/PHYTO-99-12-1346.

Gottwald, T. R. (2010). Current epidemiological understanding of citrus Huanglongbing. Annual Review of Phytopathology, 48(1), 119-139. http://dx.doi.org/10.1146/ annurev-phyto-073009-114418.

Gottwald, T. R., Graça, J. V., \& Bassanezi, R. B. (2007). Citrus huanglongbing: the pathogen and its impact. Plant Health Progress, 8(1), 31. http://dx.doi.org/10.1094/ PHP-2007-0906-01-RV.

Graça, J. V. (1991). Citrus greening disease. Annual Review of Phytopathology, 29(1), 109-136. http://dx.doi. org/10.1146/annurev.py.29.090191.000545.

Halbert, S. E., \& Manjunath, K. L. (2004). Asian citrus psyllids (Sternorrhyncha: Psyllidae) and greening disease of citrus: a literature review and assessment of risk in Florida. The Florida Entomologist, 87(3), 330-353. http:// dx.doi.org/10.1653/0015-4040(2004)087[0330:ACPSP A]2.0.CO;2.

Hilf, M. E. (2011). Colonization of citrus seed coats by 'Candidatus Liberibacter asiaticus': implications for seed transmission of the bacterium. Phytopathology, 101(10), 1242-1250. http://dx.doi.org/10.1094/PHYTO-11-10-0323.

Instituto Colombiano Agropecuario - ICA. Subgerencia de Protección Vegetal. Dirección Técnica de Epidemiología y Vigilancia Fitosanitaria. (2010). 'Candidatus Liberibacter caribbeanus', una nueva especie de Liberibacter asociada a cítricos de Colombia, Sur América. Bogotá: ICA. Recuperado el 30 de marzo de 2016, de http:/www. ica.gov.co/getattachment/da3cea77-f5d2-4e44-a40f990a37a $7 \mathrm{e} 78 \mathrm{~d} / \% \mathrm{E} 2 \% 80 \% 98 \mathrm{Candidatus-Liberibacter-}$ caribbeanus $\%$ E2\%80\%99,-una-nueva-e.aspx

Irey, M. (2010). Experiences of One Florida grower with the management of huanglongbing: success over time. In Memorias del $2^{\circ}$ Taller Internacional sobre el Huanglongbing y el Psílido Asiático de los Cítricos y 2nd International Workshop on citrus huanglongbing and the asian citrus psyllid. Mérida: SAGARPA.

Kim, J. S., Sagaram, U. S., Burns, J. K., Li, J. L., \& Wang, N. (2009). Response of sweet orange (Citrus sinensis) to 'Candidatus Liberibacter asiaticus' infection: microscopy and microarray analyses. Phytopathology, 99(1), 50-57. http://dx.doi.org/10.1094/PHYTO-99-1-0050.

Knapp, J. L., Halbert, S., Lee, R., Hoy, M., Clark, R., \& Kesinger, M. (2014). Asian citrus psyllid and citrus greening disease. Gainesville: UF/IFAS. Recuperado el 30 de marzo de 2016, de http://ipm.ifas.ufl.edu/ AgriculturalIPM/asian.shtml

Liao, H. L., \& Burns, J. K. (2012). Gene expression in Citrus sinensis fruit tissues harvested from huanglongbinginfected trees: comparison with girdled fruit. Journal of Experimental Botany, 63(8), 3307-3319. http://dx.doi. org/10.1093/jxb/ers070.

Mafra, V., Martins, P. K., Francisco, C. S., RibeiroAlves, M., Freitas-Astúa, J., \& Machado, M. A. (2013). Candidatus Liberibacter americanus induces significant reprogramming of the transcriptome of the susceptible citrus genotype. BMC Genomics, 14, 247.

Manjunath, K. L., Halbert, S. E., Ramadugu, C., Webb, S., $\&$ Lee, R. F. (2008). Detection of 'Candidatus Liberibacter asiaticus' in Diaphorina citri and Its importance in the management of citrus huanglongbing in Florida. Phytopathology, 98(4), 387-396. http://dx.doi.org/10.1094/ PHYTO-98-4-0387.

Manjunath, K. L., Ramadugu, C., Castaneda, A., Diaz, J. E., Peñaranda, E. A., Chen, J., Duan, Y. P., Halbert, S. E., \& Lee, R. F. (2015). Report of Candidatus Liberibacter caribbeanus, a new citrus- and psyllid-associated Liberibacter from Colombia, South America. In Proceedings of the Annual Meeting of American Phytopathological Society (p. 101). St. Paul: American Phytopathological Society.

Martinelli, F., Uratsu, S. L., Albrecht, U., Reagan, R. L., Phu, M. L., Britton, M., Buffalo, V., Fass, J., Leicht, E., 
Zhao, W., Lin, D., D’Souza, R., Davis, C. E., Bowman, K. D., \& Dandekar, A. M. (2012). Transcriptome profiling of citrus fruit response to huanglongbing disease. $P L O S$ One, 7(5), e38039. http://dx.doi.org/10.1371/journal. pone. 0038039 .

Orozco-Santos, M., Robles-González, M. M., VelázquezMonreal, J. J., Manzanilla-Ramírez, M. Á., HernándezFuentes, L. M., Manzo-Sánchez, G., \& Nieto-Ángel, D. (2013). Manejo integrado de las principales plagas y enfermedades en limón mexicano y limón Persa. In IX Simposio Internacional Citricola y ler Simposio Internacional sobre HLB en Cítricos Ácidos (pp. 113171). México: INIFAP.

Robles-García, P. (2012). Protocolo para establecer áreas regionales de control del huanglongbing y el psílido asiático de los cítricos (ARCOs) (60 pp.). Ciudad de México: Dirección de Protección Fitosanitaria, SENASICA, SAGARPA.

Robles-González, M. M., Velázquez-Monreal, J. J., \& Manzanilla-Ramírez, M. A., Orozco-Santos, M., FloresVirgen, R., \& Medina-Urrutia, V. M. (2010). Diseminación del huanglongbing HLB en la zona productora de limón mexicano del estado de Colima. In Ier Simposio Nacional sobre Investigación para el Manejo del Psílido Asiático de los Cítricos y el Huanglongbing en México (pp. 93-97). Monterrey: CONACYT-INIFAP.

Robles-González, M. M., Velázquez-Monreal, J. J., Manzanilla-Ramírez, M. Á., Orozco-Santos, M., MedinaUrrutia, V. M., López-Arroyo, J. I., \& Flores-Virgen, R. (2013). Síntomas del huanglongbing (HLB) en árboles de limón mexicano [Citrus aurantifolia (Christm) Swingle] y su dispersión en el estado de colima, México. Revista Chapingo Serie Horticultura, 19(1), 15-31. http://dx.doi. org/10.5154/r.rchsh.2012.01.005.

Servicio de Información Agroalimentaria y Pesquera - SIAP. Secretaría de Agricultura, Ganadería, Desarrollo Rural,
Pesca y Alimentación - SAGARP. (2015). Producción agrícola: cíclicos y perenes 2014. Ciudad de México: SIAP.

Servicio de Información Agroalimentaria y Pesquera - SIAP. Secretaría de Agricultura, Ganadería, Desarrollo Rural, Pesca y Alimentación - SAGARP. (2018). Producción agrícola: cíclicos y perenes 2017. Ciudad de México: SIAP.

Servicio Nacional de Sanidad, Inocuidad y Calidad Agroalimentaria - SENASICA. Vigilancia Epidemiológica Fitosanitaria. Sistema Integral de Referencia para la Vigilancia Epidemiológica Fitosanitaria - SIRVEF. (2018). Vigilancia del huanglongbing. Ciudad de México: SENASICA, SAGARPA.

Stover, E., Shatters Junior, R., McCollum, G., Hall, D. G., \& Duan, Y. (2010). Evaluation of Candidatus Liberibacter asiaticus titer in field-infected trifoliate cultivars: preliminary evidence for HLB resistance. Proceedings of the Annual Meeting of the Florida State Horticultural Society, 123, 115-117.

Wang, N., \& Trivedi, P. (2013). Citrus huanglongbing: a newly relevant disease presents unprecedented challenges. Phytopathology, 103(7), 652-665. http://dx.doi.org/10.1094/ PHYTO-12-12-0331-RVW.

Zheng, Z. L., \& Zhao, Y. (2013). Transcriptome comparison and gene co-expression network analysis provide a systems view of citrus response to 'Candidatus Liberibacter asiaticus' infection. BioMed Central Genomics, 14(27), 1-21.

Recibido: Mayo 21, 2018 Aceptado: Oct. 22, 2018

Cómo citar: Robles González, M. M., Orozco Santos, M., Manzanilla Ramírez, M. A., Velázquez Monreal, J. J., Medina Urrutia, V. M., \& Sanches Stuchi, E. (2018). Experiencias con huanglonbing en limón Mexicano en el Estado de Colima, México. Citrus Research \& Technology, 39, e1039. https://doi. org/10.4322/crt.16518. 